\title{
Application of information and communication technology in radiological practices: a cross-sectional study among radiologists in \\ Ghana
}

\author{
Emmanuel K M Edzie', Klenam Dzefi-Tettey², Philip N Gorleku', Ewurama A Idun³, Bernard Osei ${ }^{4}$, Obed Cudjoe $^{5}$, Abdul $^{1}$ \\ Raman Asemah', Henry Kusodzi ${ }^{1}$ \\ 1 Department of Medical Imaging, School of Medical Sciences, College of Health and Allied Sciences, University of Cape Coast, Cape Coast, Ghana, 2 \\ Department of Radiology, Korle Bu Teaching Hospital, Accra, Ghana, ${ }^{3}$ Department of Radiology, 37 Military Hospital, Accra, Ghana, ${ }^{4}$ African Institute \\ for Mathematical Science, Ghana, Accra, Ghana, ${ }^{5}$ Department of Microbiology and Immunology, School of Medical Sciences, College of Health and \\ Allied Sciences, University of Cape Coast, Cape Coast, Ghana \\ Keywords: radiological practices, application, information communication technology, ghana \\ https://doi.org/10.29392/001c.13060
}

\section{Journal of Global Health Reports}

Vol. 4, 2020

\begin{abstract}
Background
There is an inadequate number of radiologists in Ghana whose distribution are skewed in favour of urban areas, creating a huge service gap with the few radiologists overburdened with work. The only way to bridge this service gap while increasing numbers of radiologists by training is from the application of information communication technology (ICT), hence this study.
\end{abstract}

\section{Methods}

This was a cross-sectional questionnaire administered study conducted between 16th 18th May 2019 during the annual general meeting of the Ghana Association of Radiologists involving 46 consented radiologists. The questionnaire investigated the available ICT tools, imaging modalities, the degree of usage and impacts of ICT on radiologists work output.

\section{Results}

The most available modalities were digital. 13.0\% had teleradiology available, but 32.6\% were familiar with it. All radiologists indicated improvement in work output with ICT. The time taken to report a case reduced from 1-2 hours to 10-30 minutes with ICT. Majority did not communicate professional issues via e-mails. $95.7 \%, 13.0 \%$ and $10.9 \%$ of radiologists used WhatsApp, Facebook and Twitter respectively to communicate radiological information. Online research provided significant information for their radiological reporting.

\section{Conclusion}

The topmost four imaging modalities readily available were Digital Ultrasound, Digital Radiography, Computed Tomography scan and Magnetic Resonance Imaging. The availability of and familiarity with teleradiology was low in radiological practices. The reporting time per case and work output of radiologists were improved with ICT. The two most common social media platforms used by radiologists were WhatsApp and Facebook.

Information Communication Technology (ICT) has become a very important part of health care delivery and management processes such as teaching, communication, storage and retrieval of medical or health information. This has positively affected how students are taught, how patients are managed and the training of health workers among other things. ${ }^{1}$ The application of ICT is a collective term for all computer-based systems in diagnostic imaging. ${ }^{2}$

Modalities serve as a collective term for all equipment used to produce diagnostic images. These imaging modalities rely mostly on sophisticated and advanced ICT tech- nologies to capture radiological images in digital format. The most frequently used imaging modalities were Computed Radiography/mammography, Computed Tomography (CT) scan, Digital ultrasound (DU) and Magnetic Resonance Imaging (MRI). ${ }^{2}$

Picture Archiving and Communication System (PACS) is the electronic storage of multimodal data, including imaging studies, reports and patients' identification information. ${ }^{3}$ Electronic Patients Record (EPR) is a system that acts as an electronic repository of medical and administrative information related to patients. Radiology Information Sys- 
tems (RIS) covers systems supporting administrative tasks such as accounting, scheduling, lab administrations and reporting. The RIS is usually integrated with PACS. The integration level and method may vary. However, images can be opened in PACS simultaneously with patient data and the description of the examination in RIS. PACS may also duplicate information from RIS, for efficiency purposes. ${ }^{2}$

Before RIS and PACS existed, radiologists' interpretations were available only on paper. Radiologic images were created, stored, shared, and interpreted on film. ${ }^{4}$ This has been the practice in most developing countries in Africa and in other parts of the world until recent developments and the breakthrough of innovations in ICT in the health sector.

Digital Imaging and Communication in Medicine (DI$\mathrm{COM}$ ) is the standard for communication and management of medical imaging information and related data. ${ }^{5}$ Most of the available commercial software for manipulation of DICOM images are expensive by themselves or distributed with expensive medical machinery. The MicroDicom DI$\mathrm{COM}$ viewer is equipped with the most common tools for manipulation of DICOM images and it has an intuitive user interface, free for use and accessible to everyone. ${ }^{6}$ Amide's a Medical Image Data Examiner (AMIDE) has been developed as a user-friendly, open-source software tool for displaying and analysing multimodality volumetric medical images. ${ }^{7}$

With the advent of internet and informatics innovations, the limits of imaging informatics have been pushed beyond traditional borders through the introduction of new technologies, such as the World Wide Web, wireless connectivity, and more recently, the social media networks. These new technologies have significantly improved radiology practice and delivery of radiological services to clients. ${ }^{8}$

Teleradiology, according to a study done in Belgium was defined as an "electronic transmission of radiological images from one geographical location to another for the purposes of interpretation and consultation". 8 Although teleradiology might be considered to be a form of telemedicine, teleradiology should be regarded as a medical act because the images need to be interpreted by a medical specialist. Therefore teleradiology is part of the medical specialty of radiology. Teleradiology has become an integral part of the workflow in many radiology departments for various reasons, the most prevalent of which being the shortage of radiologists, or otherwise reduced operational capacities and increasing workloads. Today, teleradiology has many purposes worldwide, ranging from services for experts or second opinions to international commercial diagnostic reading services.

Although many radiologists are already using social media, a large number of them are still unaware of the wide spectrum of useful information and interaction available via social media and of the added value these platforms can bring to daily practice. For many, the risk of mixing professional and private data by using social media creates a feeling of insecurity, which still holds radiologists back from using them. ${ }^{9}$

Currently, Ghana has about 56 radiologists. This gives a ratio of one radiologist to about 553,571 people in relation to the current estimated population of about 31 million people. ${ }^{10}$ The recommended mean number of radiologists for Western Europe is 12 radiologists per 100,000 people.
Germany has 12 radiologists per 100,000 and the USA has $10-12$ per $100,000 . .^{11}$

Ghana has 0.181 radiologists per 100,000 (1.81 per $1,000,000$ people) and Nigeria has 0.231 per 100,000 (2.31 per $1,000,000$ people). ${ }^{12}$ This means that Africa generally has very low numbers of radiologists to serve their population including Ghana. These few radiologists in Ghana are spread across eight regions out of the 16 regions in the country. The distributions of these radiologists are skewed towards urban-southern part depriving the remaining eight regions of none, creating a huge service gap.

A lot is known about the introduction and application of ICT in the medical field in the developed world, but not a lot of work has been done to find out how these new technologies have impacted the medical field in developing African countries like Ghana. The previous studies done in Ghana on ICT use in the medical setting indicated that neither of these studies specifically focused on the application of ICT in radiological practices. ${ }^{13,14}$ This study therefore, aimed to determine the level of utilization or application of information and communication technology in radiological practices among Ghanaian radiologists.

\section{METHODS}

\section{STUDY SITE AND DESIGN}

This was a qualitative, cross-sectional study with the use of well-structured pretested questionnaires. The study employed primary data collected between $16^{\text {th }}-18^{\text {th }}$ May, 2019 during the annual general meeting of the Ghana Association of Radiologists which was held in Ada, Dangbe East District, Greater Accra region. This study was conducted in the Cape Coast Teaching Hospital.

The questionnaire contains the following; age, gender, availability of imaging modalities and ICT tools, different software employed in the imaging modalities, duration for reporting per case, online research for information on the patient's ailment and social media usage among radiologists.

The pretested questionnaires were self-administered to investigate the application and utilization of ICT with the available imaging modalities among radiologists. The following specific objectives were also examined:

- To determine the imaging modalities available in radiological practices in Ghana.

- To determine the availability and assess their familiarity with ICT tools in radiological practices and whether any associations exist between them and the years of practice.

- To ascertain the effect of ICT usage on work output of radiologists.

- To investigate whether ICT had an effect on the duration of radiological reporting.

- To find out the level of usage of social media in radiological practices.

\section{SAMPLING}

The official number of radiologists in Ghana was difficult to obtain from the Ghana Medical and Dental Council and 
Table 1. Distribution of available and familiar ICT tools in radiological practices*

\begin{tabular}{|c|c|c|c|c|c|}
\hline & ICT Tools Av & & & ICT Tools Fa & \\
\hline Variable & Frequency & Percentages (\%) & Variable & Frequency & Percentages (\%) \\
\hline PACS & & & PACS & & \\
\hline Yes & 29 & 63.0 & Yes & 36 & 78.3 \\
\hline No & 17 & 37.0 & No & 10 & 21.7 \\
\hline RIS & & & RIS & & \\
\hline Yes & 18 & 39.1 & Yes & 24 & 52.2 \\
\hline No & 28 & 60.9 & No & 22 & 47.8 \\
\hline EPR & & & EPR & & \\
\hline Yes & 18 & 39.1 & Yes & 25 & 54.3 \\
\hline No & 28 & 60.9 & No & 21 & 45.7 \\
\hline Teleradiology & & & Teleradiology & & \\
\hline Yes & 6 & 13.0 & Yes & 15 & 32.6 \\
\hline No & 40 & 87.0 & No & 31 & 67.4 \\
\hline
\end{tabular}

PACS - Picture Archiving and Communication System, RIS - Radiology Information Systems, EPR - Electronic Patients Record

*Source: field data, 2019.

the Ministry of Health $(\mathrm{MOH})$, but from the unpublished records of the Ghana Association of Radiologists, there were 56 registered radiologists in the whole of Ghana as at the time of this study. Out of the total, 46 radiologists took part in the study excluding four authors of this article who are also practising radiologists. The convenience sampling technique (administering to all the available-consented radiologists) was deployed for the data collection. This sampling technique was employed because using any other technique would have caused data inaccessibility.

\section{STATISTICAL ANALYSIS}

The information obtained from the questionnaire was entered using SPSS (SPSS Inc. Chicago, IL version 20.0) software for windows. The data was analysed using frequencies, percentages, and presented in appropriate tables and charts. A non-parametric test known as Wilcoxon Signed Rank Test was used to test the null hypothesis that there is no statistical difference in the average time for radiological reporting per case prior and after ICT usage. Also Chisquare test for independence was conducted to establish the association between the years of practice and the familiarity with and the availability of ICT tools in radiological practices in Ghana. The level of significance was specified at 0.05 .

\section{ETHICAL CONSIDERATION}

Ethical clearance to conduct the study was obtained from the Cape Coast Teaching Hospital ethical review committee. Informed consent was obtained from each radiologist prior to the administration of the questionnaires.

\section{RESULTS}

\section{THE DEMOGRAPHIC CHARACTERISTICS}

A total of 46 questionnaires were distributed to all the radiologists present and all (100\%) were returned and recorded. The average age of the radiologists was 43 years with a minimum and maximum age of 32 and 60 years respectively. The median age was 42 years old. There were 31 males and 15 female respondents.

\section{IMAGING MODALITIES AVAILABLE IN RADIOLOGICAL PRACTICES}

All the radiologists had Digital Ultrasound available to them. $84.8 \%, 82.6 \%$, 60.9\%, 54.3\% and $43.5 \%$ had Digital Radiography/Mammography, CT scan, MRI, Computed Radiography and Conventional Analogue Radiography available respectively in their practices.

$63.0 \%, 39.1 \%, 39.1 \%$, and $13.0 \%$ of the radiologists had PACS, EPR, RIS and Teleradiology respectively as the ICT tools available. While $78.3 \%, 54.3 \%$ and $52.2 \%$ of the respondents were familiar with PACS, EPR and RIS respectively. However, familiarity with software was lowest in Teleradiology with $32.6 \%$ of respondents (Table 1 ).

An association done to explore the relationship between the years of practice and the availability of and familiarity with ICT tools revealed no association at a confidence level of $95 \%$ (Table 2).

$58.7 \%$ of the radiologists employed the use of DICOM in their practices, but $41.3 \%$ did not. Most of the respondents 91.3\% and 73.9\% did not use MicroDicom and AMIDE tools respectively. Software usage was not on a daily basis as only $56.5 \%$ of radiologists reported usage on a daily basis, $26.1 \%$ used them one to six times in a week, $10.9 \%$ used them only occasionally with $6.5 \%$ who never used any software at all (Table 3). 
Table 2. A cross-tabulation of the number of years in radiological practice against ICT tools' availability and familiarity*

\begin{tabular}{|c|c|c|c|c|c|c|c|c|}
\hline \multirow[b]{2}{*}{ ICT Tools } & \multicolumn{6}{|c|}{ Number of years in radiological practice } & \multirow[t]{2}{*}{$x-2$} & \multirow[t]{2}{*}{$P$-value } \\
\hline & $<1$ year & 1-2years & 3-4years & 5-6years & 7-8years & 8years > & & \\
\hline \multicolumn{9}{|c|}{ Availability: } \\
\hline \multicolumn{9}{|l|}{ PACS: } \\
\hline Yes & $1(3.4 \%)$ & $1(3.4 \%)$ & $\begin{array}{l}7 \\
\text { (24.1\%) }\end{array}$ & $\begin{array}{l}3 \\
(10.3 \%)\end{array}$ & $\begin{array}{l}4 \\
(13.8 \%)\end{array}$ & $\begin{array}{l}13 \\
(44.8 \%)\end{array}$ & 4.116 & 0.533 \\
\hline No & $1(5.9 \%)$ & $\begin{array}{l}2 \\
(11.8 \%)\end{array}$ & $1(5.9 \%)$ & $\begin{array}{l}3 \\
(17.6 \%)\end{array}$ & $\begin{array}{l}2 \\
(11.8 \%)\end{array}$ & $8(47.1 \%)$ & & \\
\hline \multicolumn{9}{|l|}{ RIS: } \\
\hline Yes & $0(0.0 \%)$ & $0(0.0 \%)$ & $\begin{array}{l}3 \\
(16.7 \%)\end{array}$ & $\begin{array}{l}2 \\
(11.1 \%)\end{array}$ & $1(5.6 \%)$ & $\begin{array}{l}12 \\
(66.7 \%)\end{array}$ & 9.266 & 0.099 \\
\hline No & $2(7.1 \%)$ & $\begin{array}{l}3 \\
(10.7 \%) \\
\end{array}$ & $\begin{array}{l}5 \\
(17.9 \%) \\
\end{array}$ & $\begin{array}{l}4 \\
(14.3 \%)\end{array}$ & $\begin{array}{l}5 \\
(17.9 \%) \\
\end{array}$ & $9(32.1 \%)$ & & \\
\hline \multicolumn{9}{|l|}{ EPR: } \\
\hline Yes & $0(0.0 \%)$ & $\begin{array}{l}2 \\
(11.1 \%)\end{array}$ & $\begin{array}{l}5 \\
(27.8 \%)\end{array}$ & $\begin{array}{l}2 \\
(11.1 \%)\end{array}$ & $\begin{array}{l}2 \\
(11.1 \%)\end{array}$ & $7(38.9 \%)$ & 5.164 & 0.396 \\
\hline No & 2 (7.1\%) & $1(3.6 \%)$ & $\begin{array}{l}3 \\
(10.7 \%) \\
\end{array}$ & $\begin{array}{l}4 \\
(14.3 \%)\end{array}$ & $\begin{array}{l}4 \\
(14.3 \%) \\
\end{array}$ & $\begin{array}{l}14 \\
(50.0 \%) \\
\end{array}$ & & \\
\hline \multicolumn{9}{|c|}{ Teleradiology: } \\
\hline Yes & $0(0.0 \%)$ & $0(0.0 \%)$ & $\begin{array}{l}1 \\
(16.7 \%)\end{array}$ & $0(0.0 \%)$ & $0(0.0 \%)$ & $5(83.3 \%)$ & 6.542 & 0.257 \\
\hline No & $2(5.0 \%)$ & $3(7.5 \%)$ & $\begin{array}{l}7 \\
(17.5 \%)\end{array}$ & $\begin{array}{l}6 \\
(15.0 \%)\end{array}$ & $\begin{array}{l}6 \\
(15.0 \%)\end{array}$ & $\begin{array}{l}16 \\
(40.0 \%)\end{array}$ & & \\
\hline \multicolumn{9}{|c|}{ Familiarity: } \\
\hline \multicolumn{9}{|l|}{ PACS: } \\
\hline Yes & $1(2.8 \%)$ & $1(2.8 \%)$ & $\begin{array}{l}8 \\
(22.2 \%)\end{array}$ & $\begin{array}{l}5 \\
(13.9 \%)\end{array}$ & $\begin{array}{l}4 \\
(11.1 \%)\end{array}$ & $\begin{array}{l}17 \\
(47.2 \%)\end{array}$ & 8.083 & 0.152 \\
\hline No & $\begin{array}{l}1 \\
(10.0 \%)\end{array}$ & $\begin{array}{l}2 \\
(20.0 \%)\end{array}$ & 0 (0.0\%) & $\begin{array}{l}1 \\
(10.0 \%)\end{array}$ & $\begin{array}{l}2 \\
(20.0 \%)\end{array}$ & $4(40.0 \%)$ & & \\
\hline \multicolumn{9}{|l|}{ RIS: } \\
\hline Yes & $1(4.2 \%)$ & $0(0.0 \%)$ & $\begin{array}{l}3 \\
(12.5 \%)\end{array}$ & $\begin{array}{l}4 \\
(16.7 \%)\end{array}$ & 2 (8.3\%) & $\begin{array}{l}14 \\
(58.3 \%)\end{array}$ & 8.315 & 0.140 \\
\hline No & $1(4.5 \%)$ & $\begin{array}{l}3 \\
(13.5 \%)\end{array}$ & $\begin{array}{l}5 \\
(22.7 \%)\end{array}$ & $2(9.1 \%)$ & $\begin{array}{l}4 \\
(18.2 \%)\end{array}$ & $7(31.8 \%)$ & & \\
\hline \multicolumn{9}{|l|}{ EPR: } \\
\hline Yes & 0 (0.0\%) & 2 (8.0\%) & $\begin{array}{l}5 \\
(20.0 \%)\end{array}$ & 2 (8.0\%) & $\begin{array}{l}3 \\
(12.0 \%)\end{array}$ & $\begin{array}{l}13 \\
(52.0 \%)\end{array}$ & 5.151 & 0.398 \\
\hline No & 2 (9.5\%) & $1(4.8 \%)$ & $\begin{array}{l}3 \\
(14.3 \%)\end{array}$ & $\begin{array}{l}4 \\
(19.0 \%)\end{array}$ & $\begin{array}{l}3 \\
(14.3 \%)\end{array}$ & $8(38.1 \%)$ & & \\
\hline \multicolumn{9}{|c|}{ Teleradiology: } \\
\hline Yes & $0(0.0 \%)$ & $0(0.0 \%)$ & $\begin{array}{l}3 \\
(20.0 \%)\end{array}$ & $2(13.3 \%)$ & $1(6.7 \%)$ & $9(60.0 \%)$ & 5.774 & 0.329 \\
\hline No & 2 (6.5\%) & $3(9.7 \%)$ & $\begin{array}{l}5 \\
(16.1 \%)\end{array}$ & $4(12.9 \%)$ & $\begin{array}{l}5 \\
(16.1 \%)\end{array}$ & $\begin{array}{l}12 \\
(38.1 \%)\end{array}$ & & \\
\hline
\end{tabular}

PACS - Picture Archiving and Communication System, RIS - Radiology Information Systems, EPR - Electronic Patients Record

* $\chi-2$ was used to examine the relationship between years of practice and the availability of and familiarity with ICT tools in radiological practices. $P \leqslant 0.05$ is statistically significant.

\section{IMPROVEMENTS IN WORK OUTPUT WITH THE APPLICATION OF ICT.}

All the respondents (100\%) reported that applications of ICT improved their work output in various ways. $32.6 \%$ re- ported faster and better conveniences in reporting with ICT applications. $19.6 \%$ reported provision of clearer and more quality images with better manoeuvrability. Also, $17.4 \%$ had improvement in their turnaround time, $15.2 \%$ reported improvement in their ability to store and retrieve images with 
Table 3. Distribution of software used in radiological practices*

\begin{tabular}{lcc}
\hline Variable & Frequency & Percentages $(\%)$ \\
\hline MicroDicom: & & \\
\hline Yes & 4 & 8.7 \\
\hline No & 42 & 91.3 \\
\hline AMIDE: & & 26.1 \\
\hline Yes & 12 & 73.9 \\
\hline No & 34 & \\
\hline DICOM: & & 58.7 \\
\hline Yes & 27 & 41.3 \\
\hline No & 19 & \\
\hline Software usage: & & 56.5 \\
\hline Everyday & 26 & 26.1 \\
\hline $1-6$ times a week & 12 & 10.9 \\
\hline Only on certain occasions & 5 & 6.5 \\
\hline None & 3 & \\
\hline
\end{tabular}

AMIDE - A Medical Image Data Examiner, DICOM- Digital Imaging and Communication in Medicine *Source: field data, 2019.

Table 4. Distribution showing duration of reports per case prior and post ICT usage*

\begin{tabular}{|c|c|c|c|c|c|c|}
\hline Variable & & \multicolumn{2}{|c|}{ Frequency } & \multicolumn{3}{|c|}{ Percentages (\%) } \\
\hline \multicolumn{7}{|l|}{ Prior to ICT Usage: } \\
\hline 1-10 minutes & & \multicolumn{2}{|c|}{2} & \multicolumn{3}{|c|}{4.4} \\
\hline $10-30$ minutes & & \multicolumn{2}{|c|}{18} & \multicolumn{3}{|c|}{39.1} \\
\hline 1-2 hours & & \multicolumn{2}{|c|}{19} & \multicolumn{3}{|c|}{41.3} \\
\hline$>2$ hours & & \multicolumn{2}{|c|}{7} & \multicolumn{3}{|c|}{15.2} \\
\hline \multicolumn{7}{|l|}{ Post ICT Usage } \\
\hline $1-10$ minutes & & \multicolumn{2}{|c|}{19} & \multicolumn{3}{|c|}{41.3} \\
\hline $10-30$ minutes & & \multicolumn{2}{|c|}{27} & \multicolumn{3}{|c|}{58.7} \\
\hline 1-2 hours & & \multicolumn{2}{|c|}{0} & \multicolumn{3}{|c|}{0.00} \\
\hline$>2$ hours & & \multicolumn{2}{|c|}{0} & \multicolumn{3}{|c|}{0.00} \\
\hline \multicolumn{7}{|c|}{ Wilcoxon Signed Rank Test comparing duration of reports per case prior and post ICT Usage } \\
\hline \multirow[t]{2}{*}{ Variable } & \multicolumn{3}{|c|}{ Ranks } & Mean & \multicolumn{2}{|l|}{ Test Statistic } \\
\hline & Negative & Positive & Ties & & Z & $\mathrm{P}$ \\
\hline Prior to ICT Usage & 40 & 0 & 6 & 2.67 & 5.826 & 0.000 \\
\hline Post ICT Usage & & & & 1.59 & & \\
\hline
\end{tabular}

ICT - Information Communication Technology*Wilcoxon Signed Rank Test showing differences in the average reporting time per case prior and post ICT usage. $P \leqslant 0.05$ is statistically significant.

ICT, while $15.2 \%$ indicated the flexibility of working from different locations with the use of ICT.

Majority (41.3\%) responses indicated averagely 1-2 hours to provide reports per case prior to the use of ICT. $58.7 \%$ of the radiologists reported that it took averagely 10-30 minutes to prepare reports per case after the use of ICT. A Wilcoxon Signed Rank Test also showed; duration for a report after the use of ICT in radiology practice was lower, with a mean of 1.59 than the duration before with mean of 2.67. There was statistical evidence to reject the null hypothesis and conclude that there is a difference between the time taken by radiologists to prepare radiological reports prior to ICT usage and after the use of ICT (Table 4).

Majority of the respondents had internet access through 


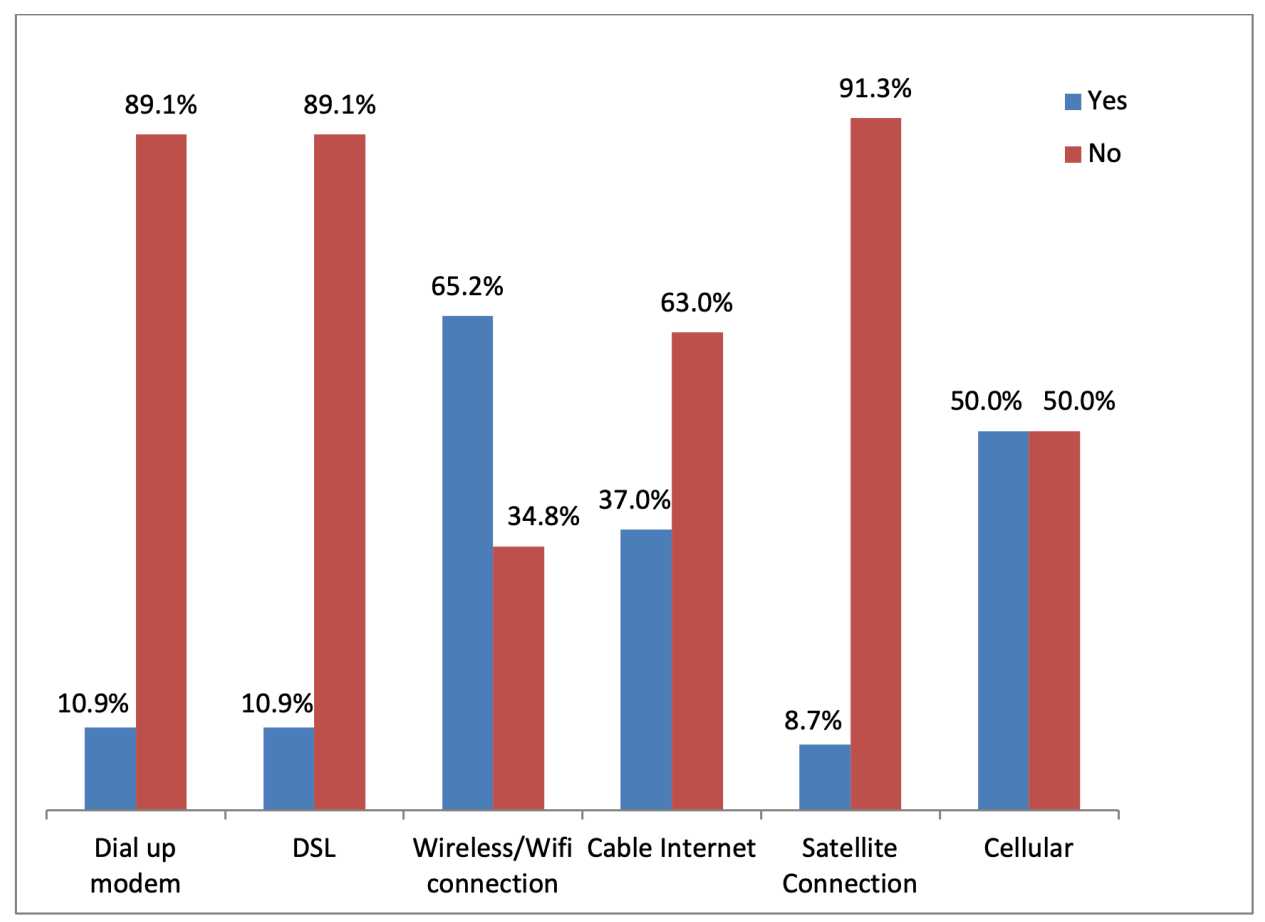

Figure 1. Mode of access to the internet by radiologists.

wireless and cellular connectivity (Figure 1).

\section{THE USE OF E-MAILS TO COMMUNICATE RADIOLOGICAL FINDINGS}

All the radiologists had e-mail addresses, but the majority of them did not communicate (63\%) with patients/clinicians via e-mails.

95.7\% of the radiologists used WhatsApp in communicating radiological information to clinicians and patients. Fewer than $4.5 \%$ of respondents used Viber, Wechat, Instagram and Skype to communicate in their practices. However, $13.0 \%$ and $10.9 \%$ of the respondents used Facebook and Twitter respectively. All the radiologists did not use the other social media networks such as Telegram, and LinkedIn at all in their practices (Figure 2).

Out of 46 radiologists, $69.6 \%$ strongly agreed and $30.4 \%$ agreed that online research helped to provide information for writing a report (Figure 3).

\section{MAJOR CHALLENGES FACED IN RADIOLOGICAL PRACTICES BY RADIOLOGISTS}

The most common challenge in radiological practices in Ghana was an unstable internet and network availability in $50 \%$ of responses. $19.6 \%$ being frequent power failures and $10.9 \%$ for systems (computer) failures. Lack of expertise to fix systems was $8.7 \%$ and lack of frequent maintenance was $6.5 \%$. Only $4.3 \%$ did not have any major challenges.

\section{OVERCOMING MAJOR CHALLENGES IN RADIOLOGICAL PRACTICES.}

The major challenges with the application of ICT in radiological practices can be overcome by ensuring stable and re- liable internet connectivity, provision of trained on-site engineers and technicians, back-up power plan, provision of workshops on new tools available and provision of regular maintenance were reported as $34.8 \%, 21.7 \%, 15.2 \%, 13.0 \%$ and $10.9 \%$ respectively. $4.3 \%$ did not offer any solution.

\section{HOW IMPACTFUL IS ICT ON RADIOLOGICAL PRACTICES.}

$60.9 \%$ of the radiologists indicated that the impact of ICT applications in radiological practices was excellent with the few (2.2\%) indicating that ICT had a fair impact on their practices.

\section{DISCUSSION}

This study showed that the digital modalities are more available than the semi digital and analogue types in Ghana. It implies that Ghanaian radiological practices have transitioned from analogue to digital systems. A report by the National Health Service (England) from the Diagnostic Imaging Dataset statistical released in November, $2018^{15}$ also found similar results, even though in Ghana we found that digital ultrasound was the commonest modality available while digital radiography was reported as the commonest in England.

The majority $(78.3 \%)$ of the respondents were familiar with PACS, but $63.0 \%$ had it available (Table 1). A finding in Canada indicated PACS systems availability of $76 \%$ in 38 sites and that of the United States also showed that majority (70.7\%) have been using PACS for more than 1 year to interpret $75-100 \%$ of cases. ${ }^{16,17}$ Both results agree with our findings. Teleradiology is the least available ICT tool in radiological practices, with a percentage availability of $13.0 \%$ and familiarity of $32.6 \%$. The healthcare sector in 


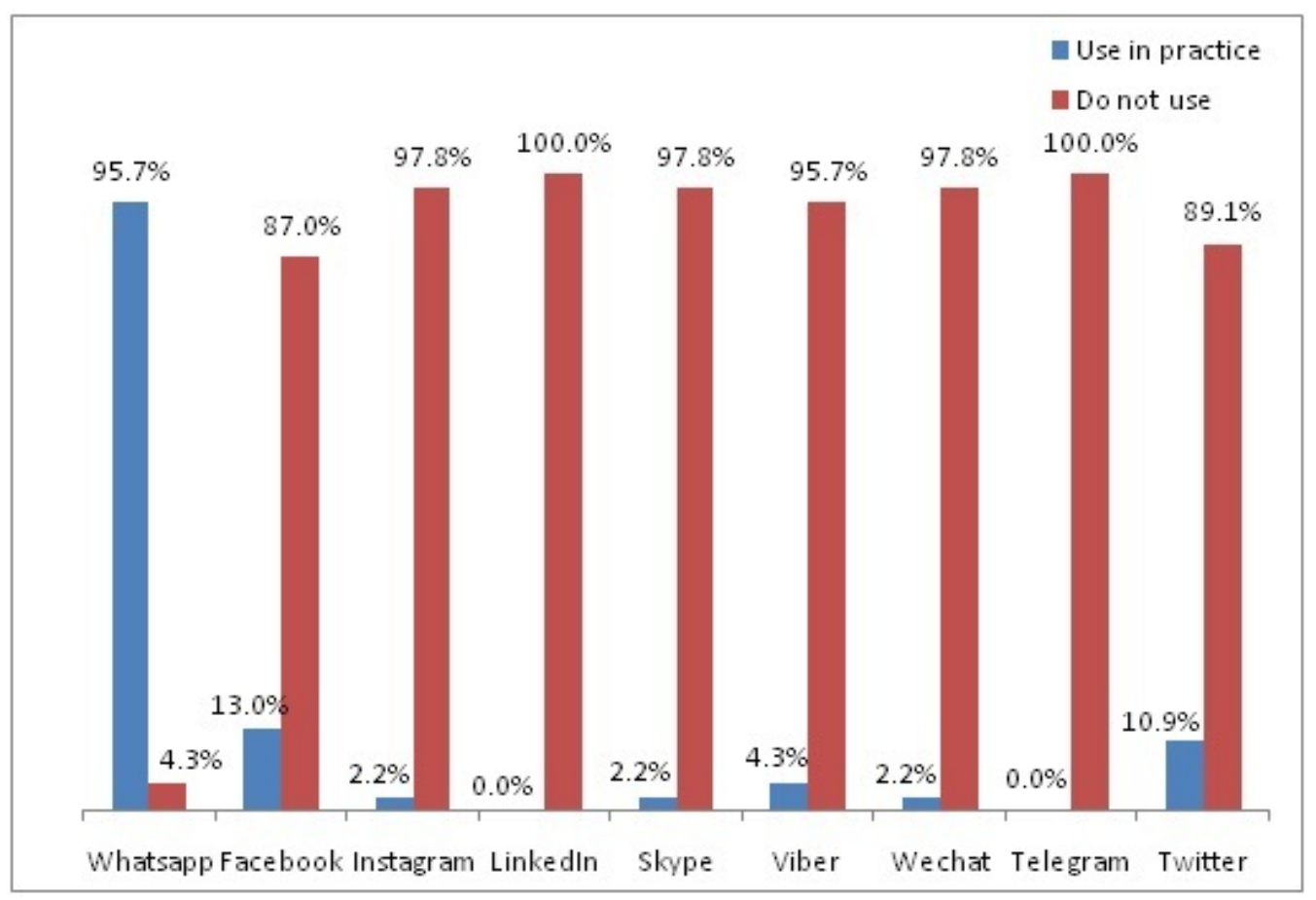

Figure 2. Distribution of social media network use in radiological practices.

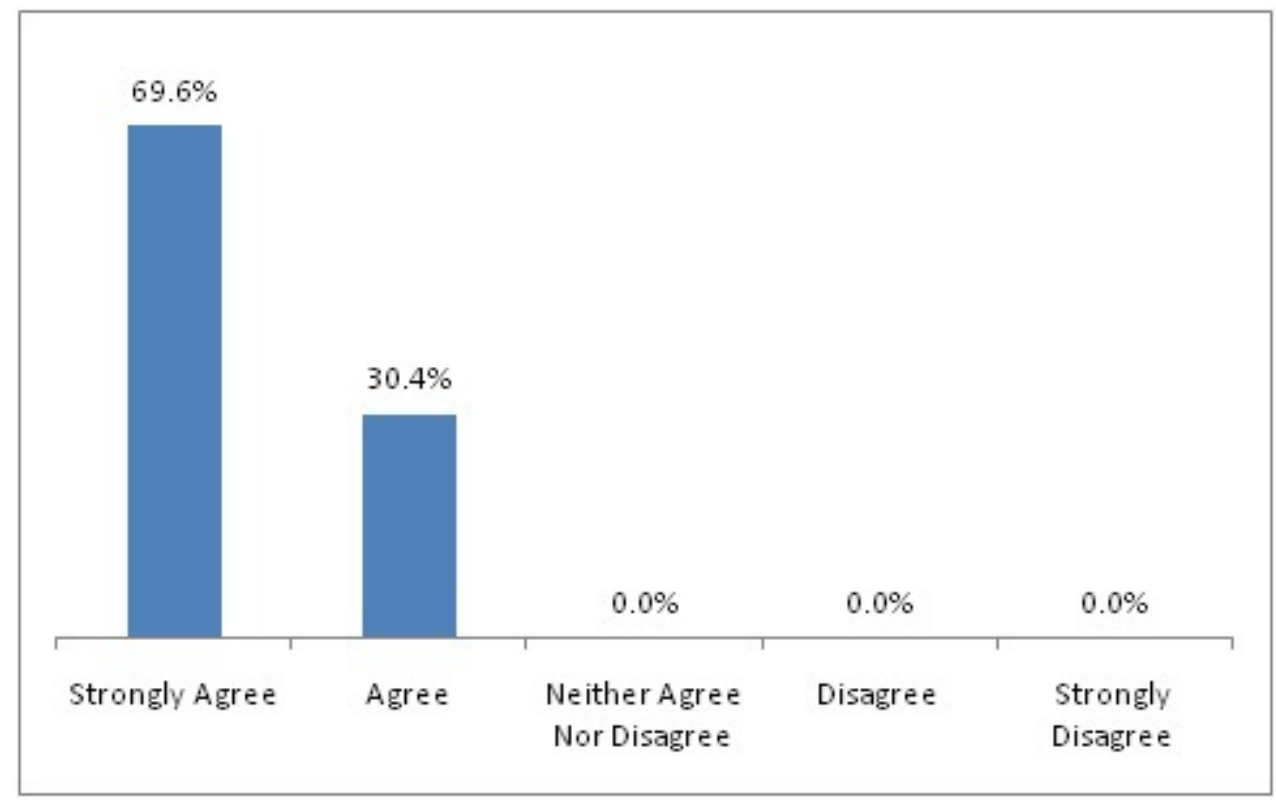

Figure 3. Distribution of online research use to diagnose patients' ailments.

Ghana is not fully connected within itself or to any nationwide network system which is a necessary prerequisite for any ICT strategy; hence telemedicine/teleradiology facilities and other advanced telecommunication facilities are low. ${ }^{18}$ Our study also found that there was no association between the duration of practice and the availability of and familiarity with ICT tools (Table 2).

The use of other software like AMIDE and MicroDicom were low except for DICOM which had a high percentage usage of $58.7 \%$. One major reason that may have accounted for this situation may be the complexity and sophisticated nature of most software that come along with most imaging modalities deployed in radiological practices.

Most of the radiologists (56.5\%) reported using these software on daily basis and the rest did not use the software daily with $6.5 \%$ who never used them. This may be partly due to the fact that many of these software were pre-installed in the various digital imaging modalities and those who never used them are likely to be using analogue systems. 
From this study, the parameters indicating work output of radiologists like ensuring faster and more conveniences in reporting, provision of clear and quality images and better manoeuvrability, turnaround time, ability to store and retrieve images for references and ability to work from different locations were all improved after the application of ICT. This finding is consistent with the study conducted in Belgium that reported integration of DICOM with dedicated plug-ins allowed radiologists to view clear-quality images with better manoeuvrability on internet connected computers with web browsers. ${ }^{8}$ The findings from Gunn AJ et al. ${ }^{19}$ also reported faster reporting and turnaround times in agreement with our findings.

The duration of radiological reporting per case significantly reduced from 1-2 hours to 10-30 minutes after the application of ICT. This is worthy of note because the reduction in reporting time will lead to provision of faster diagnoses which will lead to faster treatment, ultimately increasing patients' outcome and satisfaction. Reiner et al. ${ }^{20}$ also reported $16.2 \%$ reduction in overall time taken by radiologists for reporting soft-copy interpretation for radiological imaging modalities such as CT scan compared to that of the film similar to the reduction in the reporting time with ICT usage in our study. This is also corroborated by a study in the USA which reported decreases in reporting times, decreases in examination times and increases in the number of studies with the use of technological advances. ${ }^{19}$

This study showed that all the radiologists (100\%) had email addresses with the majority of them accessing the internet through wireless and cellular connectivity (Figure 1). Most of the respondents did not use electronic mail to communicate radiological information to clinicians and patients; this may be because sending such information may not be secured via such media in order to maintain the patient's confidential records. The non-usage of e-mails professionally by radiologists may be as a result of fear of software security breaches especially in healthcare and the fact that no framework has been outlined for dealing with the patient's data confidentiality and protection in Ghana as reported by the Ministry of Health of Ghana (18). It is possible that with a bit of effort to address the issues concerning data protection of patients, a stable and reliable network and internet connectivity; almost all radiologists in Ghana will use online resources to improve their practices.

The majority (95.7\%) of the radiologists use WhatsApp in their practices (Figure 2). This is similar to what was reported in Saudi Arabia by Alanzi et al. (21) that found WhatsApp to be the most frequently used social media in the radiology department at Johns Hopkins Aramco Healthcare in $59.6 \%$ of responses. This can enable radiologists to work distantly from where the images were taken, and therefore can help in bridging the service gap due to low numbers and poor distribution of radiologists in Ghana. However, this may not be the best, as the security of patient information sent through this medium may not be guaranteed. However, most of the radiologists did not adequately use or did not use at all the other social media platforms such as (Facebook, Twitter, Viber, Instagram, Skype, Wechat, LinkedIn and Telegram) (Figure 2). This is contrary to Ranschaert et al. (9) which showed by far Facebook was the most popular social media platform among physicians/radiologists to share radiological findings followed by LinkedIn, Pinterest, Instagram and Twitter for professional usage.

All the radiologists agreed that online research helped to solve problems relating to patients' ailments, and also helped to provide reliable reports, which is also supported by a study conducted in England in 2006 which emphasized how often searching with Google led to the correct diagnoses and found that Google searches revealed the correct diagnoses in the majority of cases (22).

The major challenges with the application of ICT in radiological practices in Ghana were unstable internet and network availability in the majority (50\%) of responses, with frequent power failures, systems (computer) failures, lack of expertise to fix systems and lack of frequent maintenance were other challenges. These corroborated with the study done in Ghana which reported frequent power failure by the Electricity Company of Ghana and lack of maintenance culture as another problem. This Ghanaian study also reported lack of corrective and preventive maintenance culture of ICT equipment even in government agencies (13). These challenges are no different from the ones reported by the Ministry of Health of Ghana (18), which stated that there is inadequate network availability/networking with unstable and unreliable internet connectivity at the national, regional and district levels.

According to the participants in this study, the challenges in radiological practices can be overcome by ensuring stable and reliable internet connectivity, provision of trained on-site engineers and technicians, back-up power plan, provision of workshops on new tools available and provision of a regular maintenance.

The majority of the radiologists indicated that the impact of ICT on their practices was excellent, very good and good in $97.8 \%$ of cases. This finding needs to be consolidated by further exposing radiologists to new and updated ICT innovations regularly in their practices.

Since there are countless numbers of ICT tools and software in medical imaging, many of which are not available in our setting and hence this study could not have looked at all these tools, which is a notable limitation of this study.

\section{CONCLUSIONS}

We conclude that Digital Ultrasound, Digital Radiography (including mammography), CT scan and MRI were the top four imaging modalities readily available and utilised in radiological practices in Ghana. The availability of teleradiology in Ghana was low and familiarity with the use of teleradiology was also low. There was a significant reduction in radiological reporting times with the application of ICT. There were improvements in the work output among the radiologists in several areas of their practices with the application of ICT. Radiologists communicated radiological findings over WhatsApp to clinicians and mostly used online research for patients' ailments. The ICT usage by the radiologists was affected greatly by unstable internet and network connectivity, frequent power failures, the computer systems failures, poor maintenance culture and lack of expertise in the management of available ICT tools.

From the above conclusions, we recommend that, the $\mathrm{MOH}$ in Ghana should implement policies to ensure data 
protection, improvement in ICT security systems and development of nationwide ICT infrastructure to enable the full utilisation of ICT in radiological practices.

Acknowledgments: The authors are grateful to all the radiologists who responded to the questionnaires to make this study possible and successful.

Funding: No external funding secured.

Authorship contributions: EKME, KD, PNG, EAI, BO, OC, ARA and HK made substantial contributions to conception, literature searches, design, the acquisition of data, analysis, interpretation of the data, drafting, revision and approval of the manuscript for publication.

Competing interests: The authors completed the Unified Competing Interest form at www.icmje.org/coi disclosure.pdf (available upon request from the corresponding author), and declare no conflicts of interest.

\section{Correspondence to:}

Emmanuel Kobina Mesi Edzie

Department of Medical Imaging

University of Cape Coast

PMB

Cape Coast

Ghana

emmanuel.edzie@ucc.edu.gh 


\section{REFERENCES}

1. Olorundare S. Utilization of information and communication technology (ICT) in curriculum development, implementation and evaluation. Information communication technology in the service of education. 2007:11-33.

\section{Hurlen P. Introducing Information and} Communication Technology to Radiologists: Impacts on Process and Outcome (Dissertation on Internet). Oslo: University of Oslo; 2011. http://urn.nb.no/UR N:NBN:no-28424.

3. Reporting SR. PACS, SRR and the future of radiology. UMOJ. 2011;80:22.

4. Kohli M, Dreyer KJ, Geis JR. Rethinking radiology informatics. AJR Am J Roentgenol. 2015;204:716-720. doi:10.2214/AJR.14.13840

5. http://dicom.nema.org/medical/dicom/current/out put/chtml/part01/chapter 1.html\#sect 1.1. Accessed December 20, 2019.

6. http://www.microdicom.com/dicom-viewer/overvie w.html. Accessed December 20, 2019.

7. Loening AM, Gambhir SS. AMIDE: A free software tool for multimodality medical image analysis. $\mathrm{Mol}$ Imaging. 2003;2:131-137. doi:10.1162/153535003322 $\underline{556877}$

8. Ranschaert ER. The Impact of Information Technology on Radiology Services: An Overview. Journal of the Belgian Society of Radiology. 2016;100(1). doi:10.5334/ibr-btr.1184

9. Ranschaert ER, van Ooijen PM, Lee S, Ratib O, Parizel PM. Social media for radiologists: An introduction. Insights into Imaging. 2015;6:741-752. doi:10.1007/s13244-015-0430-0

10. World Population Review. Ghana Population. 2020. https://worldpopulationreview.com/countries/g hana-population/. Accessed January 6, 2020.

11. GE Health Partners. Workforce Review: Radiology. Aligning Demand and Capacity in a Changing Health Care Environment 2018. https://emea.gehealthcarepa rtners.com/images/pdfs/Rapid-Review--Radiology-W orkforce-Review-FINAL.pdf. Accessed December 24, 2019.
12. Kawooya MG. Training for rural radiology and imaging in sub-saharan Africa: Addressing the mismatch between services and population. J Clin Imaging Sci. 2012;2:37. doi:10.4103/2156-7514.97747

13. Achampong EK. The state of information and communication technology and health informatics in Ghana. OJPHI. 2012;4(2). doi:10.5210/ojphi.v4i2.4191

14. Afarikumah E. Electronic health in Ghana: Current status and future prospects. Online Journal of Public Health Informatics. 2014;5:230. doi:10.5210/oj phi.v5i3.4943

15. NHS. Diagnostic Imaging Dataset Statistical Release. NHS England NHS Improvement. 2019. http s://www.england.nhs.uk/statistics/wp-content/upload s/sites/2/2019/03/Provisional-Monthly-Diagnostic-Im aging-Dataset-Statistics-2019-03-21-1.pdf. Accessed December 20, 2019.

16. Lapinsky SE, Holt D, Hallett D, Abdolell M, Adhikari NK. Survey of information technology in intensive care units in Ontario, Canada. BMC Med Inform Decis Mak. 2008;8:5. doi:10.1186/1472-694 $\underline{7-8-5}$

17. Gutierrez AJ, Mullins ME, Novelline RA. Impact of PACS and voice-recognition reporting on the education of radiology residents. Journal of Digital Imaging. 2005;18:100-108. doi:10.1007/s10278-004-1 $\underline{878-5}$

18. Ministry of Health. Health Sector ICT Policy and Strategy-Ghana. 2005. https://www.ghanahealthservi ce.org/downloads/Health\%20Sector\%20ICT\%20Polic y\%20and\%20Strategy.pdf. Accessed December 24, 2019.

19. Gunn AJ, Mangano MD, Choy G, Sahani DV. Rethinking the role of the radiologist: Enhancing visibility through both traditional and nontraditional reporting practices. RadioGraphics. 2015;35:416-423. doi:10.1148/rg.352140042

20. Reiner BI, Siegel EL, Hooper FJ, Pomerantz S, Dahlke A, Rallis D. Radiologists' productivity in the interpretation of CT scans: A comparison of PACS with conventional film. American Journal of Roentgenology. 2001;176:861-864. doi:10.2214/ajr.17 $\underline{6.4 .1760861}$ 